\title{
Orchestrating Large Organizational Change in Primary Care: The Veterans' Health Administration Experience Implementing a Patient-Centered Medical Home
}

\author{
Gordon Schectman, $\mathrm{MD}^{1,2}$ and Richard Stark, $M D^{3}$ \\ 'Office of Patient Care Services (Primary Care Services), Veterans Health Administration, Washington, DC, USA; ${ }^{2}$ Milwaukee VA Medical \\ Center, Milwaukee, WI, USA; ${ }^{3}$ Office of Operations and Management (Primary Care Operations), Veterans Health Administration, \\ Washington, DC, USA.
}

J Gen Intern Med 29(Suppl 2):S550-1

DOI: $10.1007 / \mathrm{s} 11606-014-2828-7$

(C) Society of General Internal Medicine 2014

$\mathrm{T}$ he Veterans Health Administration (VHA) has been on an evolving journey to achieve a system-wide approach to care that enables the patient-centered medical home to serve as the focal point of a medical neighborhood, closely integrating primary care, mental health, specialty care, and inpatient care, and spanning the continuum of care from health to palliative care. The journey began in the closing years of the 20th century with a forward thinking paradigm that perceived health care from the context of one large integrated system based upon team-based care with the foundation centered within a well-constructed primary care team. Over the past decade, VHA has accelerated its progression from a hospital-based system to one that has primary care as its foundation.

Since establishing this vision, VHA growth has been rapid. The Veterans Health Administration is now the largest integrated health care system in the US. VHA has almost 1,000 sites of care and salaried staff care for over 5.3 million primary care patients. More than $50 \%$ of care is provided in community-based outpatient clinics, ranging from small single practitioner clinics to larger outpatient multispecialty facilities. VA Medical Centers also range from smaller facilities to large tertiary care hospitals. The Computerized Patient Record System (CPRS), which has been in use since the 1990s, serves to unify, standardize, and coordinate care delivery throughout the system. Patient empanelment is tracked and monitored using nationally developed software that facilitates continuity and consistency across the VHA system.

Current VA leadership has reaffirmed this commitment to primary care and has encouraged new initiatives designed to further transform the way care is provided. This VHA Primary Care transformation, based on the patient centered medical home, is called the Patient Aligned Care Team (PACT). In this new model, providers require enhanced

Published online April 9, 2014 skills in order to effectively collaborate with patients to help manage their care and share in decisions. These core skills include cultural competency, motivational interviewing, proficiency in managing disagreements, as well as other enhanced communication skills such as active listening and using healing therapeutic language. Proficiency in managing change also contributes to meaningful experiences for patients and staff. Core behaviors are enabled by personal mastery of the skills that can ultimately lead to healthy partnerships.

The VHA's integrated health care system allows for care to be delivered via many modalities. The traditional face-toface appointment visit remains the chief way of delivering personalized care. But in addition there are group visits and education sessions, both in person and via videoconferencing. Secure messaging is also used to communicate between patients and team members, and tele-consultation is utilized to bring specialty care closer to veterans who may be located at some distance from the medical center. Specialty consultation can be performed electronically via chart review, obviating the need for patients to travel. Home telehealth enables extensive monitoring of vital health care processes (such as blood pressure, glucose, pulse oximetry, and weight) to be brought into the patient's home and communicate directly back to the health care team. Mobile applications are being developed to enable patients and their teams to communicate wherever they are.

The first step in organizing this change was a baseline assessment of resources and processes. Staffing allocation was examined and resources made available to bring personnel levels up to recommended standards. In April 2010 , the VHA began to augment primary care teams to ensure at least four full-time health care professionals per panel of patients. Team roles and functions were specified, and clear expectations for the whole team were established. Additional team members were specified to provide added support, including social workers, clinical pharmacy specialists, nutritionists, health behaviorists, and others.

Once team staffing was in place, training of all members of the team could begin. The curriculum was developed by multiple workgroups representing many aspects of VHA's 
transformation initiatives, drawing on the resources of the entire system to formulate the teachings that would advance the entire organization along the identified path to patientcenteredness. Given the scope of the task, the underlying principle was to provide training via several modalities in order to reach the 20,000 core staff across the country that make up PACTs. Top leadership in primary care frequently served as faculty at training events in order to assure delivery of the correct message as well as to demonstrate commitment to the changes at the highest levels of the organization. The first major training event was an IHI-like Learning Collaborative, consisting of over 250 teams participating in 6 learning sessions and work periods over 18 months. Those who were not able to participate in the collaborative were scheduled to attend briefer interactive sessions locally. Coaches were identified to help teams remain on track with changes. In addition, consultation teams were deployed to assist sites that requested additional help and instruction in change techniques. Complicating the training effort were several pauses due to new restrictions on travel and meetings for VHA staff, delaying and forcing reconfiguration of the training to a mostly virtual format. In addition to national training efforts, regularly scheduled national conference calls bring together communities of practice to share successes and discuss challenges and barriers. National leadership participates in these calls as well as in other teleconferences, video conferences and webinars to directly answer questions and address concerns. Some of the results of these training initiatives are included in this supplement.

Implementation of the PACT model is monitored through a number of metrics that have been collected into a PACT "Compass," which enables national system-wide sharing of data related to PACT implementation. These include metrics related to panel management, patient engagement and satisfaction, continuity, access, care coordination, and clinical improvement. A primary feature of this compass is the ability for PACTs to drill down from national data all the way to their own individual teams, allowing performance improvement to be monitored at the team level. Teams showing evidence of excellence are identified, recognized nationally, and invited to share their strong practices for a national VHA clinical audience.

VHA also provided resources for five demonstration laboratories to study various aspects of PACT implementation in order to guide the process and inform both leadership and the field of those practices that work best in the context of VHA practice. Staff satisfaction as well as adaptation to change is a particular focus of these centers, and they have provided valuable feedback on processes and practices related to implementation. One of the important lessons identified has been the significance of leadership buy-in and involvement in successful implementation. Other important themes tied to effective implementation are highlighted in this supplement and include topics as varied as the importance of initial PACT education and training efforts; the evaluation of relationships among team function, PACT processes, and desirable outcomes; and an assessment of barriers and challenges to successful implementation.

Within the first 2 years following the launch, $80 \%$ of the highest performing PACTs were located in only $30 \%$ of VA facilities - those where facility and local primary care leadership demonstrated their active support.

At least two key lessons may be learned from VHA's approach to PACT implementation. First, in any large-scale reform, cultural transformation is paramount and requires engaged, persistent leadership as well as thoughtful, longitudinal training of all staff. Second, choosing the right metrics to evaluate innovations should be approached sensitively. Frontline clinicians initially clamored for metrics to provide definition for goals and objectives - but subsequently were critical when they were used to assess performance. We learned that metrics are more supportable when they flow from the patient-centered vision - and therefore incorporate patients' feedback wherever possible - spurring performance improvement activities that are focused on understanding and addressing patients' needs and concerns, while providing a fulcrum for collaboration of all team members. These metrics not only provide the inspiration and tools to fuel the search for knowledge and understanding, but ultimately also improve the final product.

Although VHA's transformation to the PACT model is still in its early stages, the papers presented in this supplement provide encouragement that culture change in a large organization is not only possible, but if effectively monitored and managed, can also improve the quality of care.

Corresponding Author: Gordon Schectman, MD; Office of Patient Care Services (Primary Care Services), Veterans Health Administration, 810 Vermont Avenue, Washington, DC 20420, USA (e-mail: Gordon.schectman@va.gov). 